DOI 10.1007/s10559-016-9832-3

Cybernetics and Systems Analysis, Vol. 52, No. 2, March, 2016

\title{
ERRATUM 1
}

To the article "Fuzzy Object-Oriented Dynamic Networks. II" by D. A. Terletskyi and A. I. Provotar, Vol. 52, No. 1, pp. 38-45, January, 2016.

The abbreviations NRM and NRMs were erroneously used in the article. These abbreviations should be replaced everywhere by KRM and KRMs, respectively. 